\title{
Risk Factors and Outcome of Acute Kidney Injury after Isolated CABG Surgery: a Prospective Cohort Study
}

\author{
Shahram Amini ${ }^{1}$, MD, FCCM; Mona Najaf Najafi², MD, Seyedeh Parissa Karrari ${ }^{1}$, MD; Mohammadghasem Etemadi \\ Mashhadi ${ }^{3}$, MD; Sahereh Mirzaei ${ }^{4}$, RN, BSN, MSc; Mohammad Abbasi Tashnizi ${ }^{5}$, MD; Ali Asghar Moeinipour ${ }^{5}$, MD; \\ Hamid Hoseinikhah ${ }^{5}$, MD; Mathias Hossain Aazami ${ }^{3}$, MD; Mahdieh Jafari ${ }^{1}$, MD
}

DOI: 10.21470/1678-9741-2017-0209

\begin{abstract}
Background: Acute kidney injury (AKI) is a frequent event after cardiac surgery with increased mortality and morbidity. We explored frequency, risk factors, and associated morbidity and mortality of AKI after isolated coronary artery bypass grafting (CABG) surgery at a single institution.

Methods: All consecutive adults undergoing CABG surgery from March 2013 to October 2016 were assessed for development and severity of AKI based on Acute Kidney Injury Network (AKIN) criteria. The patients were also investigated regarding their need for renal replacement therapy (RRT), predictive risk factors, and associated outcomes, including duration of mechanical ventilation, mortality, intensive care unit (ICU) and hospital length of stay.

Results: Of 1737 patients in the study, 275 (15.8\%) developed AKI. Twenty-five $(\mathbf{1 2 . 8 \%})$ cases required RRT. Patients with AKI had longer ventilation time, ICU and hospital length of stay $(P<0.001)$. Mortality rates were $28(10.2 \%)$ and $22(1.5 \%)$ in patients with and
\end{abstract}

without AKI, respectively $(P<0.001)$. There was a strong association between advanced age (aOR=1.016, 95\% Cl=1.002-1.030, $P=0.028)$, diabetes (aOR=1.36, 95\% Cl=1.022-1.809, $P=0.035)$, on-pump surgery $(\mathrm{aOR}=2.63,95 \% \mathrm{Cl}=1.543-4.483, P<0.001)$, transfusion of more than 1 unit of red blood cells $(\mathrm{aOR}=2.154,95 \% \mathrm{Cl}=1.237-3.753$, $P=0.007)$, and prolonged mechanical ventilation and development of AKI (aOR=2.697, 95\% Cl=1.02407.071, $P<0.001)$. AKI was seen less frequently in those with opium abuse (aOR=0.613, 95\% $\mathrm{Cl}=0.409-0.921, P=0.018$ ).

Conclusion: We demonstrated that advanced age, diabetes, on-pump surgery, red blood cell transfusion, and prolonged mechanical ventilation were independent positive risk factors for the development of AKI after isolated CABG while opium abuse was a protective factor.

Keywords: Acute Kidney Injury. Coronary Artery Bypass. Treatment Outcome.

\begin{tabular}{ll} 
Abbreviations, acronyms \& symbols \\
\hline AKI & $=$ Acute kidney injury \\
AKIN & $=$ Acute Kidney Injury Network \\
CABG & $=$ Coronary artery bypass grafting \\
CPB & $=$ Cardiopulmonary bypass \\
EGFR & $=$ Estimated glomerular filtration rate \\
IABP & $=$ Intra-aortic balloon pump \\
ICU & $=$ Intensive care unit \\
RBC & $=$ Red blood cells \\
ROC & $=$ Receiver operating characteristic \\
RRT & $=$ Renal replacement therapy
\end{tabular}

'Department of Anesthesiology and Critical Care, Mashhad University of Medical Sciences, Mashhad, Iran.

${ }^{2}$ Clinical Research Unit, Faculty of Medicine, Mashhad University of Medical Sciences, Mashhad, Iran.

${ }^{3}$ Department of Anesthesia, Faculty of Medicine, Mashhad University of Medical Sciences, Mashhad, Iran.

${ }^{4}$ Department of Biobehavioral Sciences, College of Nursing, University of Illinois at Chicago, Chicago, USA.

${ }^{5}$ Department of Cardiac Surgery, Mashhad University of Medical Sciences, Mashhad, Iran.

This study was carried out Mashhad University of Medical Sciences, Mashhad, Iran.

Financial Support Mashhad University of Medical Sciences n. 930357.

\section{INTRODUCTION}

Acute kidney injury (AKI) is a common complication after cardiac surgery. The incidence has been reported from $6.7 \%$ to $39 \%[1-4]$, based on definition of AKI. It is associated with increased morbidity and mortality that is further increased in more severe stages of $A \mathrm{KI}^{[5-7]}$. It also increases intensive care unit (ICU) and hospital length of stay ${ }^{[2,8]}$, and use of resources ${ }^{[2]}$. Renal replacement therapy (RRT) is required in $1.6 \%$ to $7.7 \%$ after cardiac surgery, which may further increase the mortality rate in these patients ${ }^{[9,10]}$.

Several perioperative risk factors have been reported as predictors of $\mathrm{AKI}$ associated with cardiac surgery, including

\section{No conflict of interest.}

Correspondence Address:

Shahram Amini

(iD) https://orcid.org/0000-0002-2785-1812

Emam Reza Hospital

Cardiac Anesthesia Research Center

Department of Anesthesiology and Critical Care

Ebne Sina Street, Mashhad, Iran

E-mail:aminish@mums.ac.ir 
advanced age ${ }^{[11,12]}$, time interval of angiography and surgery ${ }^{[13]}$, blood transfusion ${ }^{[4,11,14]}$, preoperative elevated serum creatinine ${ }^{[15,16]}$, diabetes ${ }^{[17]}$, high-risk according to the EuroSCORE ${ }^{[18]}$, and use of nephrotoxic drugs. Use of cardiopulmonary bypass (CPB) has also been proposed to be a predictive factor for development of AKI after coronary artery bypass grafting $(C A B G)^{[19,20]}$. In addition to the use of prophylactic drugs ${ }^{[21]}$, knowing the predisposing factors and modifying them can help to decrease the incidence of AKI.

The objective of this study was to explore the frequency of AKI after isolated CABG at a single teaching hospital. The primary outcome was to determine the frequency of AKI. The secondary outcomes included determination of risk factors and associated outcomes, including duration of mechanical ventilation, ICU and hospital length of stay, and in-hospital mortality.

\section{METHODS}

After obtaining approval from the university's ethics committee, we recruited all consecutive adults undergoing isolated CABG from March 2013 to October 2016 at a university teaching hospital in Mashhad, Iran. Patients were excluded if they had no documented preoperative serum creatinine or expired within 24 hours.

Baseline variables included demographic parameters, preoperative hemoglobin, urea, creatinine, creatinine clearance, and any comorbidities.

Surgical characteristics including type of surgery, duration of surgery, use of CPB and intraoperative events, including excessive bleeding, life-threatening arrhythmia, and intraoperative transfusion were recorded.

All patients underwent standard anesthesia and were transferred to cardiac surgery ICU where they were under standard monitoring and meticulous attention by intensive care team including intensivists, critical care fellows, anesthesiology residents, and trained registered nurses to maintain optimal cardiopulmonary, cerebral and renal function.

The patients were assessed postoperatively for development, severity, time of onset, and duration of AKI. Urine output was not used as a criterion of AKI in our study due to the frequent use of diuretics in these patients. AKI was defined based on the
Acute Kidney Injury Network (AKIN) criteria (Table 1). Estimated glomerular filtration rate (eGFR) was calculated using CockroftGault equation. RRT was used in the case of refractory acidbase and electrolyte disorders, signs of hypervolemia or uremic encephalopathy (defined as decreased level of consciousness, assuming to be related to hyperuremia).

They were also investigated for mechanical ventilation for more than 24 hours, ICU and hospital length of stay, and in-hospital death. Patients were weaned from mechanical ventilation and were extubated according to a standardized protocol.

\section{Statistical Analysis}

Means and standard deviation were used for normal distribution variables and median and interquartile range for otherwise. Frequencies and percentages were used for categorical variables.

The Student's t-test or the Mann-Whitney test was used to compare continuous variables between patients with and without AKI. Chi-square test or Fisher's exact test was used for categorical variables.

Binary logistic regression model was constructed for assessment of independent effect of each variable on predicting the occurrence of AKI. Model goodness of fit was evaluated by the Hosmer-Lemeshow test. The receiver operating characteristic (ROC) curve was used for prediction of sensitivity and specificity of cutoff point of significant variables. Statistical significance was considered as $P$ value of $<0.05$. The statistical analysis was performed using SPSS ${ }^{\circledR}$ version 16 (IBM SPSS, Chicago, IL, USA).

\section{RESULTS}

During the study period, 1737 patients were recruited. CPB was used in 293 (16.9\%) cases. There were 1073 (61.8\%) males and 664 (38.2\%) females, with a mean age of $59.93 \pm 10.34$ years. Demographic data and surgical characteristics of patients are presented in Table 2.

AKI was seen in 275 (15.8\%) patients. Of these, 81.8\%, 8.4\% and $9.8 \%$ cases developed stage I, II, and III, respectively. The median duration of AKI was 2 days. One hundred and eighty-one

Table 1. AKIN criteria.

\begin{tabular}{l|c}
\hline Serum creatinine criteria & \multicolumn{1}{|c}{ Urine output criteria } \\
\hline Definition & \\
\cline { 1 - 2 } Increase in SCr by $0.3 \mathrm{mg} / \mathrm{dL}$ or $\geq 1.5$ times baseline within 48 hours & \\
\hline Staging & $<0.5 \mathrm{~mL} / \mathrm{kg}$ per hour for more than 6 hours \\
\hline 1: Increase in SCr by $\geq 0.3 \mathrm{mg} / \mathrm{dL}$ or $\geq 1.5-<2.0$ times baseline & $<0.5 \mathrm{~mL} / \mathrm{kg}$ per hour for more than 12 hours \\
\hline 3: Increase in SCr by $\geq 3.0$ times baseline & $<0.3 \mathrm{~mL} / \mathrm{kg}$ per hour for 24 hours or anuria for 12 hours \\
\hline SCr=serum creatinine &
\end{tabular}


Table 2. Demographic data and patients' characteristics.

\begin{tabular}{|c|c|c|c|c|}
\hline & Total & AKI & No AKI & P-value \\
\hline Age (years) & $60(53-67)$ & $61(55-70)$ & $59(53-66)$ & 0.001 \\
\hline Sex (male) & $1073(61.8)$ & $167(60.7)$ & 906 (62) & 0.74 \\
\hline Weight (kg) & $70(62-79)$ & $71(63-79)$ & $70(61-79)$ & 0.46 \\
\hline Height (cm) & $165(158-171)$ & $165(158-170)$ & $165(158-171)$ & 0.86 \\
\hline $\mathrm{BMI}$ & $25.71(23.24-28.72)$ & $25.71(23.36-29.16)$ & $25.70(23.24-28.66)$ & 0.37 \\
\hline Ml & $152(8.8)$ & $24(8.7)$ & $128(8.8)$ & $>0.99$ \\
\hline HTN & $829(47.7)$ & $140(50.9)$ & $689(47.1)$ & 0.26 \\
\hline Diabetes & $592(34.1)$ & $106(38.5)$ & $486(33.2)$ & 0.09 \\
\hline HLP & $551(31.7)$ & $90(32.7)$ & $461(31.5)$ & 0.72 \\
\hline EF & $50(40-55)$ & $50(40-55)$ & $50(40-55)$ & 0.17 \\
\hline NYHA (I/II/III/IV) & 248/951/492/46 & $33 / 148 / 85 / 9$ & 215/803/407/37 & 0.12 \\
\hline Left main or 3VD & $1117(64.3)$ & $182(66.2)$ & $935(64)$ & 0.49 \\
\hline Smoking & $223(12.8)$ & $29(10.5)$ & $194(13.3)$ & 0.24 \\
\hline Opium abuse & 335 (19.3) & $38(13.8)$ & 297 (20.3) & 0.012 \\
\hline Baseline creatinine & $1(0.9-1.2)$ & $1(0.9-1.3)$ & $1(0.9-1.2)$ & 0.27 \\
\hline Baseline GFR & $70.10(54.84-87.55)$ & $66.70(47.01-86.73)$ & $70.42(56.09-88.06)$ & 0.009 \\
\hline Date of angiography & $12(4-24)$ & $11(3-26)$ & $12(5-24)$ & 0.34 \\
\hline Use of IABP & $26(1.5)$ & $8(2.9)$ & $18(1.2)$ & 0.053 \\
\hline Transfusion of red blood cells & $73(4.2)$ & $26(9.5)$ & $47(3.2)$ & $<0.001$ \\
\hline
\end{tabular}

Data are presented as median $\pm \mathrm{IQR}$ (interquartile range) or numbers (percentage).

$3 \mathrm{VD}=3$ vessel disease; $\mathrm{AKI}=$ Acute kidney injury; $\mathrm{BMI}=$ body mass index; $\mathrm{EF}=$ ejection fraction; $\mathrm{GFR}=$ glomerular filtration rate; HLP=hyperlipidemia; HTN=hypertension; IABP=intra-aortic balloon pump; LM=left main; MI=myocardial infarction; NYHA=New York Heart Association

(65.8\%) patients recovered with a median duration of 1 day. In 94 (34.2\%) patients, AKI lasted during their hospital stay and 25 (12.8\%) patients required RRT.

AKI was seen in $193(13.4 \%)$ and 82 (28\%) cases in patients with and without $C P B$, respectively $(P<0.001)$, with the highest frequency in those undergoing on-pump beating surgery (Table 3 ).

Mortality was seen in $28(10.2 \%)$ and $22(1.5 \%)$ patients with and without $A K I$, respectively $(P<0.001)$, with $18(8 \%), 3(13 \%)$ and 7 (25.9\%) cases in those with stage I, II, and III, respectively.

The median duration of mechanical ventilation was 320 minutes (222.5-502.5), with 360 (240-675) and 315 (220-480) minutes in those with and without $\mathrm{AKI}$, respectively $(P<0.001)$.

Prolonged ventilation time (defined as ventilation over 24 hours) occurred in 11 (4\%) and 15 (1\%) patients with and without AKI, respectively $(P<0.001)$. Duration of ICU stay was $2^{[2-4]}$ days in patients with AKI and 2 (2-3) days in those without AKI $(P<0.001)$. Hospital length of stay was 7 (6-11) and 7 (6-8) days in patients with and without $\mathrm{AKI}$, respectively $(P<0.001)$.

There was an association between advanced age, drug abuse, type of surgery, use of CPB, surgical duration, use of intraaortic balloon pump (IABP), transfusion of red blood cells (RBC), and prolonged mechanical ventilation and development of AKI (Table 4). Multivariate regression analysis showed that advanced age, diabetes, use of CPB, transfusion of RBC, and prolonged mechanical ventilation were significantly associated with AKI (Table 5). In addition, opium abuse was a protective negative factor (Table 5).

\section{DISCUSSION}

Our study revealed that AKI is a common finding after isolated CABG with increased mortality and morbidity, including duration of mechanical ventilation, ICU and hospital length of stay. We also demonstrated that advanced age, diabetes, on-pump surgery, transfusion of RBC, and prolonged mechanical ventilation were independent positive risk factors for development of AKI, while drug abuse was a protective factor.

A number of mechanisms have been proposed as the etiology for development of AKI after heart surgery, including altered renal blood flow, hypoperfusion, inflammation, loss of pulsatile flow, ischemia, decreased autoregulation and nephrotoxic medications ${ }^{[9]}$. 
Table 3. Frequency and stages of acute kidney injury in different surgical techniques.

\begin{tabular}{|c|c|c|c|c|}
\hline & Off-pump & On-pump & On-pump beating & $P$-value \\
\hline Total & $1444(83.1)$ & $204(11.7)$ & 89 (1.5) & \\
\hline No AKI & 1251 (86.6) & $153(75)$ & $58(65.2)$ & \multirow{2}{*}{$<0.001$} \\
\hline AKI & $193(13.4)$ & $51(25.0)$ & $31(34.8)$ & \\
\hline AKIN 1 & $159(82.4)$ & $40(78.4)$ & $26(83.9)$ & \\
\hline AKIN 2 & $15(7.8)$ & $6(11.8)$ & $2(6.5)$ & \\
\hline AKIN 3 & $19(9.8)$ & $5(9.8)$ & $3(9.7)$ & \\
\hline RRT & $13(0.9)$ & $8(3.9)$ & $4(4.5)$ & \\
\hline
\end{tabular}

$\mathrm{AKI}=$ Acute kidney injury; AKIN=Acute kidney injury network

Table 4. Univariate analysis of risk factors associated with acute kidney injury after coronary artery bypass grafting.

\begin{tabular}{|c|c|c|c|c|}
\hline & B & OR & $95 \% \mathrm{Cl}$ & $P$-value \\
\hline \multicolumn{5}{|l|}{ Preoperative } \\
\hline Age (years) & 0.021 & 1.02 & $1.008-1.03$ & $<0.001$ \\
\hline Sex (male) & -0.052 & 0.949 & $0.729-1.24$ & 0.69 \\
\hline Diabetes & 0.231 & 1.26 & $0.965-1.644$ & 0.089 \\
\hline Drug abuse & -0.464 & 0.629 & $0.436-0.906$ & 0.013 \\
\hline Baseline GFR & -0.005 & 0.995 & $0.990-1.001$ & 0.08 \\
\hline \multicolumn{5}{|l|}{ Intraoperative } \\
\hline \multicolumn{5}{|l|}{ Surgery type (ref.: off-pump): } \\
\hline On-pump & 0.77 & 2.161 & $1.521-3.07$ & $<0.001$ \\
\hline On-pump beating & 1.243 & 3.464 & $2.183-5.497$ & $<0.001$ \\
\hline Duration of surgery & 0.003 & 1.003 & $1.001-1.005$ & 0.002 \\
\hline Use of IABP & 0.877 & 2.404 & $1.035-5.584$ & 0.041 \\
\hline \multicolumn{5}{|l|}{ Postoperative } \\
\hline Transfusion of red blood cells & 1.145 & 3.144 & $1.911-5.171$ & $<0.001$ \\
\hline Mechanical ventilation $>24$ hours & 1.391 & 4.019 & $1.826-8.848$ & 0.001 \\
\hline
\end{tabular}

$\mathrm{GFR}=$ glomerular filtration rate; $\mathrm{IABP}=$ Intra-aortic balloon pump

Our findings are in accordance with previous studies demonstrating that advanced age, diabetes, use of CPB and transfusion are independent positive risk factors for AKI.

Similar to a review by Karkouti[ ${ }^{[14]}$, we found that transfusion of RBC was associated with an increased rate of $A K I(O R=2.154)$. Decreased RBC deformability leading to small capillary obstruction, inability to handle oxygen due to deficient 2, 3-diphosphoglycerate and short lifespan of transfused RBC resulting in hemolysis and production of more free iron are the proposed mechanism of AKI associated with transfusion. However, in another study Karkouti et al. ${ }^{[22]}$ reported that this effect had been positive only in anemic patients.

We could not find an association between time interval of angiography and surgery and AKI. This is similar to findings of Ko et al. ${ }^{[23]}$, but in contrast to Hu et al. ${ }^{[13]}$. It seems that only a close succession of angiography and surgery can be associated with higher incidence of AKI after CABG. In our study, the median time interval between angiography and surgery was 12 days ${ }^{[4-24]}$.

We demonstrated a strong association between AKI and advanced age. However, the association does not seem to be clinically significant $(O R=1.016)$. This can be attributed to the younger age of our patients. Similar findings were reported by Ried et al. ${ }^{[12]}$.

There is no agreement on association of CPB and postoperative AKI. While Schopka et al. ${ }^{[24]}$ reported no protective effect of off-pump versus on-pump surgery, a meta-analysis by Seabra et al. ${ }^{[19]}$ revealed that patients undergoing off-pump CABG were $40 \%$ less affected by AKI than on-pump CABG. We 
Table 5. Multivariate analysis of risk factors associated with acute kidney injury after coronary artery bypass grafting.

\begin{tabular}{|c|c|c|c|c|}
\hline & B & OR & $95 \% \mathrm{Cl}$ & $P$-value \\
\hline \multicolumn{5}{|l|}{ Preoperative } \\
\hline Age (years) & 0.016 & 1.016 & $1.002-1.030$ & 0.028 \\
\hline Diabetes & 0.308 & 1.360 & $1.022-1.809$ & 0.035 \\
\hline Opium abuse & -0.489 & 0.613 & $0.409-0.921$ & 0.018 \\
\hline \multicolumn{5}{|l|}{ Intraoperative } \\
\hline \multicolumn{5}{|l|}{ Surgery type (ref.: off-pump): } \\
\hline On-pump & 0.651 & 1.917 & $1.307-2.811$ & 0.001 \\
\hline On-pump beating & 0.967 & 2.63 & $1.543-4.483$ & $<0.001$ \\
\hline Transfusion of red blood cells & 0.768 & 2.154 & $1.237-3.753$ & 0.007 \\
\hline \multicolumn{5}{|l|}{ Postoperative } \\
\hline Mechanical ventilation $>24$ hours & 0.99 & 2.691 & $1.024-7.071$ & $<0.001$ \\
\hline
\end{tabular}

OR=adjusted odds ratio; $95 \% \mathrm{Cl}=95 \%$ confidence interval

found that use of CPB was strongly associated with AKI with on-pump beating technique as the most common causative factor. It was not possible to perform a statistical analysis on frequency of different stages of AKI and the need for RRT among patients undergoing different surgical techniques due to their low frequency. However, there does not seem to be a significant clinical difference regarding frequency of severe AKI (defined as stage 3 of AKIN) and need for RRT among the three groups.

In line with Oezkur et al. ${ }^{[17]}$, we also noted that diabetes was an independent risk factor for AKI. Interestingly, Kocogulları et al. ${ }^{[25]}$ suggested an association between preoperative level of $\mathrm{Hb}$ $A_{1 C}$ and the development of $A K I$ in non-diabetics.

AKI was seen less commonly in patients using opium in our study. Hence, opium abuse might have a protective effect against AKI (OR=0.613, 95\% Cl=0.409-0.921). This can be attributed to possible antioxidant properties of natural opium used in our area. However, this cannot be extrapolated to synthetic opioids. As far as we know, this is the first study demonstrating such an effect and larger multicenter studies are needed to confirm this finding.

Our study had a few limitations. First, we did not investigate the changes between preoperative and intraoperative blood pressure that might affect the incidence of $A K^{[26]}$. Second, we did not use urine output as a definition for AKI since many patients received diuretics and this may affect accuracy of urine output as a variable for definition of AKI. Finally, the sample size for different techniques of CABG was not similar due to the preference and experience of different surgeons that may affect the results.

\section{CONCLUSION}

In conclusion, we found that CABG associated AKI increases mortality and morbidity. Furthermore, advanced age, diabetes, use of $\mathrm{CPB}$, transfusion of $\mathrm{RBC}$, and prolonged mechanical ventilation are independent risk factors for the development of AKI, while drug abuse is a negative risk factor.

\section{ACKNOWLEDGMENT}

We would like to thank Mrs. Farideh Golhasani and nursing staff of post cardiac surgery ICU of Emam Reza Hospital, Mashhad, Iran, for their contribution to this study.

\section{Authors' roles \& responsibilities}

SA Substantial contributions to the conception or design of the work; or the acquisition, analysis, or interpretation of data for the work; drafting the work or revising it critically for important intellectual content; agreement to be accountable for all aspects of the work in ensuring that questions related to the accuracy or integrity of any part of the work are appropriately investigated and resolved; final approval of the version to be published

MNN Analysis and interpretation of data for the work; final approval of the version to be published

SPK Acquisition of data for the work; final approval of the version to be published

MEM Acquisition of data for the work; final approval of the version to be published

SM Acquisition of data for the work; final approval of the version to be published

MAT Substantial contributions to the conception or design of the work; final approval of the version to be published

AAM Substantial contributions to the conception or design of the work; final approval of the version to be published

$\mathrm{HH}$ Substantial contributions to the conception or design of the work; final approval of the version to be published

MHA Substantial contributions to the conception or design of the work; final approval of the version to be published

MJ Acquisition of data for the work; final approval of the version to be published 


\section{REFERENCES}

1. Brown JR, Kramer RS, Coca SG, Parikh CR. Duration of acute kidney injury impacts long-term survival after cardiac surgery. Ann Thorac Surg. 2010;90(4):1142-8.

2. Dasta JF, Kane-Gill SL, Durtschi AJ, Pathak DS, Kellum JA. Costs and outcomes of acute kidney injury (AKI) following cardiac surgery. Nephrol Dial Transplant. 2008;23(6):1970-4.

3. Rosner MH, Okusa MD. Acute kidney injury associated with cardiac surgery. Clin J Am Soc Nephrol. 2006;1 (1):19-32.

4. Vellinga S, Verbrugghe W, De Paep R, Verpooten G, Janssen van Doorn K. Identification of modifiable risk factors for acute kidney injury after cardiac surgery. Neth J Med. 2012;70(10):450-4.

5. Gude D, Jha R. Acute kidney injury following cardiac surgery. Ann Card Anaesth. 2012;15(4):279-86.

6. Hudson C, Hudson J, Swaminathan M, Shaw A, Stafford-Smith M, Patel UD. Emerging concepts in acute kidney injury following cardiac surgery. Semin Cardiothorac Vasc Anesth. 2008;12(4):320-30.

7. Karkouti K, Wijeysundera DN, Yau TM, Callum JL, Cheng DC, Crowther $M$, et al. Acute kidney injury after cardiac surgery: focus on modifiable risk factors. Circulation. 2009;119(4):495-502.

8. Mao MA, Thongprayoon C, Wu Y, Tejwani V, Vela-Ortiz M, Dearani $J$, et al. Incidence, severity, and outcomes of acute kidney injury in octogenarians following heart valve replacement surgery. Int J Nephrol. 2015;2015:237951.

9. Coppolino G, Presta P, Saturno L, Fuiano G. Acute kidney injury in patients undergoing cardiac surgery. J Nephrol. 2013;26(1):32-40.

10. Perez-Valdivieso JR, Monedero P, Vives M, Garcia-Fernandez N, BesRastrollo M; GEDRCC (Grupo Español de Disfunción Renal en Cirugía Cardiaca). Cardiac-surgery associated acute kidney injury requiring renal replacement therapy. A Spanish retrospective case-cohort study. BMC Nephrol. 2009;10(1):27.

11. Freeland K, Jahromi AH, Duvall LM, Mancini MC. Postoperative blood transfusion is an independent predictor of acute kidney injury in cardiac surgery patients. J Nephropathol. 2015;4(4):121-6.

12. Ried M, Puehler T, Haneya A, Schmid C, Diez C. Acute kidney injury in septua- and octogenarians after cardiac surgery. BMC CardiovasC Disord. 2011;11(1):52.

13. Hu Y, Li Z, Chen J, Shen C, Song Y, Zhong Q. The effect of the time interval between coronary angiography and on-pump cardiac surgery on risk of postoperative acute kidney injury: a meta-analysis. J Cardiothorac Surg. 2013;8(1):178.

14. Karkouti K. Transfusion and risk of acute kidney injury in cardiac surgery. Br J Anaesth. 2012;109(suppl 1):i29-i38.
15. Chen SW, Chang CH, Fan PC, Chen YC, Chu PH, Chen TH, et al. Comparison of contemporary preoperative risk models at predicting acute kidney injury after isolated coronary artery bypass grafting: a retrospective cohort study. BMJ Open. 2016;6(6):e010176.

16. Li XH, Xiao F, Zhang SY. Investigation of risk factors of acute kidney injury after off-pump coronary artery bypass grafting and 3 years' follow-up. Beijing Da Xue Xue Bao Yi Xue Ban. 2017;49(1):131-6.

17. Oezkur M, Wagner M, Weismann D, Krannich JH, Schimmer C, Riegler C, et al. Chronic hyperglycemia is associated with acute kidney injury in patients undergoing CABG surgery: a cohort study. BMC CardiovasC Disord. 2015;15(1):41.

18. Moura EB, Bernardes Neto S-CG, Amorim FF, Viscardi RC. Correlation of the EuroSCORE with the onset of postoperative acute kidney injury in cardiac surgery. Rev Bras Ter Intensiva. 2013;25(2):233-8.

19. Seabra VF, Alobaidi S, Balk EM, Poon AH, Jaber BL. Off-pump coronary artery bypass surgery and acute kidney injury: a meta-analysis of randomized controlled trials. Clin J Am Soc Nephrol. 2010;5(10):1734-44.

20. Spunda R, Valek M, Salmay M, Prskavec T, Pecha O, Lindner J, et al. Differential impact on acute kidney injury incidence between on-and off pump coronary artery bypass grafting in octogenarians. Biomed Pap Med Fac Univ Palacky Olomouc Czech Repub. 2015;159(3):449-54.

21. Savluk OF, Guzelmeric F, Yavuz Y, Cevirme D, Gurcu E, Ogus H, et al. $\mathrm{N}$-acetylcysteine versus dopamine to prevent acute kidney injury after cardiac surgery in patients with preexisting moderate renal insufficiency. Braz J Cardiovasc Surg. 2017;32(1):8-14.

22. Karkouti K, Wijeysundera DN, Yau TM, McCluskey SA, Chan CT, Wong P-Y, et al. Influence of erythrocyte transfusion on the risk of acute kidney injury after cardiac surgery differs in anemic and nonanemic patients. Anesthesiology. 2011;115(3):523-30.

23. Ko B, Garcia S, Mithani S, Tholakanahalli V, Adabag S. Risk of acute kidney injury in patients who undergo coronary angiography and cardiac surgery in close succession. Eur Heart J. 2012;33(16):2065-70.

24. Schopka S, Diez C, Camboni D, Floerchinger B, Schmid C, Hilker M. Impact of cardiopulmonary bypass on acute kidney injury following coronary artery bypass grafting: a matched pair analysis. J Cardiothorac Surg. 2014;9(1):20.

25. Kocogulları CU, Kunt AT, Aksoy R, Duzyol C, Parlar H, Saskın H, et al. Hemoglobin A1c levels predicts acute kidney injury after coronary artery bypass surgery in non-diabetic patients. Braz J Cardiovasc Surg. 2017;32(2):83-9.

26. Kanji HD, Schulze CJ, Hervas-Malo M, Wang P, Ross DB, Zibdawi M, et al. Difference between pre-operative and cardiopulmonary bypass mean arterial pressure is independently associated with early cardiac surgery-associated acute kidney injury. J Cardiothorac Surg. 2010;5(1):71. 